PROCEEDINGS OF THE

AMERICAN MATHEMATICAL SOCIETY

Volume 137, Number 6, June 2009, Pages 1925-1932

S 0002-9939(09)09863-3

Article electronically published on February 9, 2009

\title{
SEMISIMPLICITY CRITERIA FOR IRREDUCIBLE HOPF ALGEBRAS IN POSITIVE CHARACTERISTIC
}

\author{
AKIRA MASUOKA \\ (Communicated by Gail R. Letzter)
}

\begin{abstract}
We prove that a finite-dimensional irreducible Hopf algebra $H$ in positive characteristic is semisimple if and only if it is commutative and semisimple if and only if the restricted Lie algebra $P(H)$ of the primitives is a torus. This generalizes Hochschild's theorem on restricted Lie algebras, and also generalizes Demazure and Gabriel's and Sweedler's results on group schemes in the special but essential situation with a finiteness assumption added.
\end{abstract}

\section{INTRODUCTION}

During the last two decades or so, much progress has been brought to the study of semisimple Hopf algebras; see [M], for example. Let $H$ be a finite-dimensional Hopf algebra over a field $k$ with antipode $S$. Let us consider the following conditions.

(a) $H$ is semisimple as an algebra.

(b) $H$ is cosemisimple as a coalgebra.

(c) The characteristic ch $k$ does not divide the dimension $\operatorname{dim} H$.

(d) $S$ is an involution, i.e., $S \circ S=\mathrm{id}_{H}$.

Summarizing results by Larson $[\mathrm{L}$, Larson and Radford $[\mathrm{LR}$, and Etingof and Gelaki EG], we have that (a) \& (b) $\Longleftrightarrow$ (c) \& (d) and that if $\operatorname{ch} k=0$ or $>\sqrt{d}^{\varphi(d)}$, where $d=\operatorname{dim} H$, then (a) $\Longleftrightarrow$ (b) $\Longleftrightarrow(\mathrm{d})$. Moreover, Etingof and Gelaki EG] showed that by 'lifting', most of the known results on semisimple (then necessarily cosemisimple) Hopf algebras in characteristic zero can extend to semisimple and cosemisimple Hopf algebras in positive characteristic. However, little seems to be known on those semisimple Hopf algebras in positive characteristic which are not cosemisimple. In this paper we will determine when a finite-dimensional irreducible Hopf algebras in positive characteristic is semisimple. Recall from [Sw1, Sect. 8.0] that a Hopf algebra $H$ over a field $k$ is said to be irreducible (as a coalgebra), if the coradical of $H$ equals $k$ or, equivalently, if the trivial $H$-comodule $k$ is a unique (up to isomorphism) simple $H$-comodule; this property is very opposite to cosemisimplicity. In this case the dimension $\operatorname{dim} H$, if finite, equals a power of $p$, provided $\operatorname{ch} k=p>0$; see Proposition 1.1 (1).

Received by the editors July 14, 2008.

2000 Mathematics Subject Classification. Primary 16W30.

Key words and phrases. Hopf algebra in positive characteristic, restricted Lie algebra.

(C)2009 American Mathematical Society Reverts to public domain 28 years from publication 
Throughout we work over a field $k$. Let $\bar{k}$ denote the algebraic closure of $k$. Given a finite group $G$, we let $k^{G}$ denote the dual (commutative semisimple) Hopf algebra of the group algebra $k G$. Our aim is to prove the following.

Theorem 0.1. Suppose $\operatorname{ch} k=p>0$. Let $H$ be an irreducible Hopf algebra of finite dimension. Then the following are equivalent:

(i) $H$ is semisimple.

(ii) $H$ is commutative and semisimple.

(iii) $H \otimes \bar{k} \cong \bar{k}^{G}$, where $G$ is a finite $p$-group.

(iv) The restricted Lie algebra $P(H)$ of all primitives in $H$ is a torus; see Definition 1.2 .

This generalizes Hochschild's theorem on restricted Lie algebras. This also generalizes Demazure and Gabriel's and Sweedler's results on group schemes in the special but essential situation with a finiteness assumption added. Keep the assumption $\operatorname{ch} k=p>0$. For a restricted Lie algebra $L$ of finite dimension, the restricted enveloping algebra $u(L)$ is a cocommutative irreducible Hopf algebra of finite dimension. Hochschild $[\mathrm{H}]$ proved the theorem above when $H=u(L)$. Supposing $k=\bar{k}$, Demazure and Gabriel [DG, IV, §3, 3.7] proved that a connected algebraic affine group scheme $\mathfrak{G}$ is diagonalizable if it does not have any closed subgroup scheme isomorphic to $\boldsymbol{\alpha}_{p}$. Sweedler [Sw2] proved that a connected reductive affine group scheme $\mathfrak{G}$ is necessarily abelian; see also [DG, IV, $\S 3,3.6]$, referred to as Théorème de Nagata. In the situation above, suppose that $\mathfrak{G}$ is finite, or in other words that the coordinate algebra $\mathcal{O}(\mathfrak{G})$ is finite-dimensional. Then the dual Hopf algebra $\mathcal{O}(\mathfrak{G})^{*}$ is cocommutative, and is irreducible by the connectedness assumption, whence our theorem can apply to $\mathcal{O}(\mathfrak{G})^{*}$. More precisely, the last two results with $\mathfrak{G}$ supposed to be finite are equivalent to our results, (iv) $\Rightarrow$ (iii), (i) $\Rightarrow$ (ii), respectively, with $H$ supposed to be cocommutative. It is easy to deduce those two results on $\mathfrak{G}$ in general, as Sweedler Sw2 actually did, from the results in the special case of $\mathfrak{G}$ being finite; see Remark 3.2. The author does not yet know how our theorem can extend to infinite-dimensional Hopf algebras in the dual situation.

Our proof of the theorem is quite simple, based on an elementary observation on what we call relative primitives; see the proof of our key lemma, Lemma 2.2. It does not depend on any of the three known results cited above and differs from their proofs.

\section{Preliminaries}

Let $H$ be a Hopf algebra. The coalgebra structures and the antipode are denoted by

$$
\Delta: H \rightarrow H \otimes H, \quad \Delta(h)=\sum h_{1} \otimes h_{2}, \quad \varepsilon: H \rightarrow k, \quad S: H \rightarrow H,
$$

respectively. We let $H^{+}=\operatorname{Ker} \varepsilon$ denote the augmentation ideal.

Suppose $\operatorname{dim} H<\infty$. Then, $H$ is semisimple if and only if $H \otimes \bar{k}$ is semisimple. Recall also that $H$ is commutative and semisimple if and only if $H \otimes \bar{k} \cong \bar{k}^{G}$, where $G$ is a finite group.

The subspace

$$
P(H)=\{x \in H \mid \Delta(x)=x \otimes 1+1 \otimes x\}
$$


in $H$ consisting of all primitives forms a Lie algebra with respect to the commutator $[x, y]=x y-y x$. We have

$$
P(H) \otimes \bar{k}=P(H \otimes \bar{k}) .
$$

Suppose that $H$ is irreducible. Then the coradical filtration $k=H_{0} \subset H_{1} \subset \cdots$ makes $H$ into a filtered Hopf algebra $H=\bigcup_{n>0} H_{n}$, so that the associated graded Hopf algebra gr $H=\bigoplus_{n \geq 0} H_{n} / H_{n-1}\left(H_{-1}=0\right)$ is strictly graded in the sense that gr $H(0)=k$, gr $H(1)=P(\operatorname{gr} H)$; see [Sw1, Sect. 11.2]. It is known that gr $H$ is commutative [Sw1, Theorem 11.2.5].

In what follows until the end of this section, we suppose $\operatorname{ch} k=p>0$. Given a restricted Lie algebra $L$, the restricted enveloping algebra $u(L)$ naturally forms a cocommutative irreducible Hopf algebra. It is characterized as such a Hopf algebra $H$ that is generated by $P(H)$, and satisfies $P(H)=L$. If $\operatorname{dim} L=n$, then $\operatorname{dim} u(L)=p^{n}$. In every Hopf algebra $H, P(H)$ forms a restricted Lie algebra with respect to $x^{[p]}=x^{p}$, the $p^{\text {th }}$ power in $H$, and generates a Hopf subalgebra isomorphic to $u(P(H))$.

Proposition 1.1. Let $H$ be an irreducible Hopf algebra of finite dimension.

(1) Set $L=P\left((\operatorname{gr} H)^{*}\right)$ in the dual graded Hopf algebra (gr $\left.H\right)^{*}$ of gr $H$. Then, $L$ is a positively graded restricted Lie algebra $\bigoplus_{n>0} L(n)$ generated by $L(1)$, and $u(L)=(\operatorname{gr} H)^{*}$. In particular, $\operatorname{dim} H=p^{n}$ if $n=\operatorname{dim} L$.

(2) $H$ is commutative and semisimple if and only if $H \otimes \bar{k} \cong \bar{k}^{G}$, where $G$ is a finite p-group.

(3) $H$ is commutative, semisimple and generated by $P(H)$ if and only if $u(P(H))$ $=H$ and $P(H)$ is a torus (see Definition 1.2 below) if and only if $H \otimes \bar{k} \cong \bar{k}^{G}$, where $G$ is a p-torsion finite abelian group.

Proof. (1) This follows since $(\mathrm{gr} H)^{*}$ is generated by the first component and is cocommutative by [Sw1, Theorem 11.2.5].

(2) This follows, since a finite group algebra $k G$ is local if and only if $G$ is a $p$-group.

(3) Suppose that $G$ is a finite $p$-group. Notice then that $\bar{k}^{G}$ is generated by primitives if and only if $\bar{k} G$ is commutative and $x^{p}=0$ for every element $x$ in the augmentation ideal of $\bar{k} G$ if and only if $G$ is abelian and $p$-torsion. The desired result now follows from (2) above and the next proposition, (a) $\Longleftrightarrow(\mathrm{e})$.

Definition 1.2 ( $[\mathrm{SF}$, p. 86]). Let $L$ be a restricted Lie algebra of finite dimension. $L$ is called a torus if

(1) $L$ is abelian, and

(2) every element of $L$ is semisimple in $u(L)$, i.e., generates a semisimple subalgebra.

Proposition 1.3 (Hochschild $[\mathrm{H}]$ ). For a restricted Lie algebra $L$ of finite dimension, the following are equivalent:

(a) $L$ is a torus.

(b) $L$ is abelian and is spanned by $L^{[p]}$.

(c) $L \otimes \bar{k}$ does not contain any non-zero element $z$ with $z^{[p]}=0$.

(d) $u(L)$ is semisimple.

(e) $u(L)$ is commutative and semisimple. 
Remark 1.4. To prove Theorem 0.1 we will not use this last proposition. On the contrary, the equivalences (c) $\Longleftrightarrow$ (d) $\Longleftrightarrow$ (e) follow from (the proof of) the theorem; see Remark 3.1] In particular, (c) (or (d)) implies that $L$ is abelian. Let us record here a proof of the remaining implications.

(e) $\Longrightarrow($ a). In $u(L)$, the subalgebra $k[x]$ generated by an element $x \in L$ is a Hopf subalgebra, which is semisimple if $u(L)$ is; see [Mo, 3.2.3, p.31].

(a) $\Longrightarrow$ (e). In general, the minimal polynomial of $0 \neq x \in L$ in $u(L)$ is of the form

$$
c_{0} x+c_{1} x^{p}+\cdots+c_{r-1} x^{p^{r-1}}+x^{p^{r}} \quad\left(r>0, c_{i} \in k\right),
$$

where $x, x^{p}, \cdots, x^{p^{r}}$ are linearly dependent in $L$ with $r$ minimal. The element $x$ is semisimple if and only if $c_{0} \neq 0$, in which case $x$ generates a separable subalgebra. Condition (a) implies that in the affine algebra $u(L)$, the largest separable subalgebra equals $u(L)$.

(b) $\Longleftrightarrow$ (c). We may assume that $L$ is abelian. Let $k^{1 / p}$ denote the subfield of $\bar{k}$ (including $k$ ) consisting of the $p^{\text {th }}$ roots of all elements in $k$. By the last assumption we have a map

$$
\phi_{L}: L \otimes k^{1 / p} \rightarrow L, \quad \phi_{L}\left(x \otimes c^{1 / p}\right)=c x^{[p]} .
$$

Regard $L \otimes k^{1 / p}$ as a $k$-vector space through $k \rightarrow k^{1 / p}, c \mapsto c^{1 / p}$. Then, $\phi_{L}$ is $k$-linear, and the base extension $\phi_{L} \otimes \bar{k}$ to $\bar{k}$ is identified with $\phi_{L \otimes \bar{k}}$. Therefore, (b) and (c) are both equivalent to the bijectivity of $\phi_{L}$.

\section{The space $P(H, K)$ of Relative Primitives}

Before Lemma 2.2, the characteristic ch $k$ may be arbitrary. Let $H$ be a Hopf algebra, and let $K \subset H$ be a Hopf subalgebra. We define a subspace of $H$ by

$$
P(H, K)=\left\{x \in H^{+} \mid \Delta(x)-x \otimes 1-1 \otimes x \in K \otimes K\right\},
$$

whose elements may be called relative primitives. We see that $K^{+} \subset P(H, K)$. Let

$$
a \rightarrow h=\sum a_{1} h S\left(a_{2}\right) \quad(a, h \in H)
$$

denote the conjugation by $H$ on itself. Then, $H$ is a left $H$-module under $\rightarrow$.

Proposition 2.1. (1) If $B \subset K$ is a cocommutative Hopf subalgebra, then $P(H, K)$ is a $B$-submodule of $H$ under the conjugation.

(2) Let $k\langle P(H, K)\rangle$ denote the subalgebra of $H$ generated by $P(H, K)$. Then this is a Hopf subalgebra including $K$.

(3) Suppose that $H$ is irreducible. If $H \supsetneq K$, then $P(H, K) \supsetneq K^{+}$.

Proof. (1) Since in general,

$$
\Delta(a \rightarrow h)=\sum a_{1} h_{1} S\left(a_{3}\right) \otimes\left(a_{2} \rightarrow h_{2}\right),
$$

it follows that if $a \in B$, then $\Delta(a \rightarrow h)=\sum\left(a_{1} \rightarrow h_{1}\right) \otimes\left(a_{2} \rightarrow h_{2}\right)$. This implies the assertion.

(2) This follows since one sees that the sum $k+P(H, K)$ forms a subcoalgebra of $H$ stable under the antipode.

(3) Since $H$ is irreducible, the filtered Hopf subalgebra $\bigcup_{n \geq 0} F_{n} H(\subset H)$ given by the wedge products $F_{n} H=\bigwedge^{n+1} K$ coincides with $H$; see [Mo, Sect. 5.2]. Explicitly, we have $F_{0} H=K$, and

$$
F_{1} H=\{h \in H \mid \Delta(h) \in K \otimes H+H \otimes K\} .
$$


Let $\mathcal{H}=\bigoplus_{n \geq 0} F_{n} H / F_{n-1} H\left(F_{-1} H=0\right)$ denote the associated graded Hopf algebra. As is easily seen, $F_{1} H$ is a subcoalgebra of $H$, and the natural $\left(F_{1} H, F_{1} H\right)$ bicomodule structure on $F_{1} H$ induces a $(K, K)$-bicomodule structure, say $K \otimes$

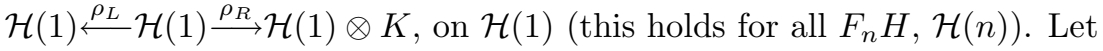

$$
\mathcal{H}(1)_{0}=\left\{u \in \mathcal{H}(1) \mid \rho_{L}(u)=1 \otimes u, \rho_{R}(u)=u \otimes 1\right\}
$$

denote the subspace in $\mathcal{H}(1)$ consisting of all coinvariants on both sides. We see that $k+P(H, K)$ is included in $F_{1} H$, and coincides with the pullback of $\mathcal{H}(1)_{0}$ along the natural projection $F_{1} H \rightarrow \mathcal{H}(1)$. Hence we have a linear surjection $P(H, K) \rightarrow$ $\mathcal{H}(1)_{0}$, whose kernel equals $K^{+}$, and which therefore induces an isomorphism

$$
P(H, K) / K^{+} \cong \mathcal{H}(1)_{0} .
$$

The assumption $K \subsetneq H$ implies $K \subsetneq \mathcal{H}$, which in turn implies $\mathcal{H}(1) \neq 0$ since the iterated coproduct induces a monomorphism $\mathcal{H}(n) \rightarrow \mathcal{H}(1)^{\otimes n}$ for each $n>0$. Since $K$ is irreducible, $\mathcal{H}(1)_{0}$ coincides with the socle of the $(K, K)$-bicomodule $\mathcal{H}(1)$ and hence is non-zero. By (2.3), this proves $P(H, K) \supsetneq K^{+}$, as desired.

Keep the situation as above. The following is a key result for proving Theorem 0.1

Lemma 2.2. Suppose $\operatorname{ch} k=p>0$ and that $k$ is perfect. Suppose $\operatorname{dim} H<\infty$ and $K \cong k^{G}$, where $G$ is a finite p-group. Suppose in addition that $H$ does not contain any non-zero primitive $z$ with $z^{p}=0$. Then, $K$ is included in the center of $k\langle P(H, K)\rangle$.

Proof. Being a finite $p$-group, $G$ has a central series, $G=G_{0} \supset G_{1} \supset \cdots \supset G_{s}=$ $\{1\}$. Thus for each $0<i \leq s, G_{i}$ is normal in $G$, and $G_{i-1} / G_{i}$ is central in $G / G_{i}$. Set $\bar{G}_{i}=G / G_{i}, B_{i}=k^{\bar{G}_{i}}$. Then we have the inclusions

$$
k=B_{0} \subset B_{1} \subset \cdots B_{s}=K
$$

of commutative Hopf algebras. Set

$$
\bar{B}_{i}=k^{G_{i-1} / G_{i}}\left(=B_{i} / B_{i} B_{i-1}^{+}\right) .
$$

Then the Hopf algebra quotient $B_{i} \rightarrow \bar{B}_{i}, b \mapsto \bar{b}$ is cocentral in the sense that

$$
\sum b_{1} \otimes \bar{b}_{2}=\sum b_{2} \otimes \bar{b}_{1} \quad\left(b \in B_{i}\right) .
$$

To prove the lemma we need to prove that $K$ trivially acts on $P(H, K)$ under the conjugation $\rightarrow($ see (2.1) $)$ or explicitly that $a \rightarrow x=\varepsilon(a) x$ for all $a \in K$, $x \in P(H, K)$. The first step is to prove that $B_{1}$ trivially acts on $P(H, K)$. Since $K$ is commutative, $B_{1}$ (and moreover $K$ ) trivially acts on $K$. Since $B_{1}$ is cocommutative, it follows by Proposition 2.1 (1) that $P(H, K)$ is a $B_{1}$-submodule of $H$. Let $x \in$ $P(H, K)$, and define

$$
\gamma_{x}:=\Delta(x)-x \otimes 1-1 \otimes x \quad(\in K \otimes K) .
$$

Notice that

$$
\gamma_{a \rightarrow x}=\varepsilon(a) \gamma_{x} \quad\left(a \in B_{1}\right) .
$$

Let $e_{g}\left(g \in \bar{G}_{1}\right)$ denote the primitive idempotents in $B_{1}=k^{\bar{G}_{1}}$, so that $e_{g}(f)=\delta_{f, g}$ $\left(f \in \bar{G}_{1}\right)$. We wish to prove

$$
e_{g} \rightarrow x=\delta_{1, g} x \quad\left(g \in \bar{G}_{1}\right) .
$$


We may rechoose $x$ so that $B_{1} \rightarrow x$ is 1 -dimensional, or in other words, so that $x \neq 0$ and

$$
e_{g} \rightarrow x=\delta_{t, g} x \quad\left(g \in \bar{G}_{1}\right)
$$

for some $t \in \bar{G}_{1}$. We aim to prove $t=1$. Suppose $t \neq 1$, on the contrary. We then see that $x$ is a primitive or $\gamma_{x}=0$. In fact we see from (2.6), (2.7) that

$$
\delta_{t, g} \gamma_{x}=\varepsilon\left(e_{g}\right) \gamma_{x}\left(=\delta_{1, g} \gamma_{x}\right) \quad\left(g \in \bar{G}_{1}\right),
$$

which tells us that $\gamma_{x}=0$ when $g=t(\neq 1)$. Notice now that $x^{p^{i}} \in P(H)$ for each $i \geq 0$. Since $\operatorname{dim} H<\infty$, the subalgebra $k[x]$ of $H$ generated by $x$ has a defining relation of the form

$$
c_{0} x+c_{1} x^{p}+\cdots+c_{r-1} x^{p^{r-1}}+x^{p^{r}}=0 \quad\left(r>0, c_{i} \in k\right) .
$$

See (1.2). We have $c_{i}^{1 / p}$ in $k$, since $k$ is perfect. We see $c_{0} \neq 0$, since otherwise,

$$
z:=c_{1}^{1 / p} x+\cdots+c_{r-1}^{1 / p} x^{p^{r-2}}+x^{p^{r-1}}
$$

would be a non-zero primitive with $z^{p}=0$. Notice $e_{g} \rightarrow x^{p^{i}}=\delta_{t^{p^{i}}, g} x^{p^{i}}$. By applying $e_{g} \rightarrow$ to (2.8), we obtain

$$
\delta_{t, g} c_{0} x+\cdots+\delta_{t^{p^{r}}, g} x^{p^{r}}=0 .
$$

Choose $t^{p^{r}}$ as $g$. By comparing (2.8) with (2.10), it then follows that $t=g=t^{p^{r}}$, which implies $t=1$, as desired.

As the second step we wish to prove that $B_{2}$ trivially acts on $P(H, K)$. Let $a \in B_{2}, x \in P(H, K)$. Then, $a \rightarrow x \in H$. By the result of the first step above, this last action factors through $\bar{B}_{2}$ so that $a \rightarrow x=\bar{a} \rightarrow x$. By (2.2) and (2.4), we see that

$$
\begin{aligned}
\varepsilon(a) \gamma_{x} & =\Delta(a \rightarrow x)-(a \rightarrow x) \otimes 1-\sum a_{1} S\left(a_{3}\right) \otimes\left(\bar{a}_{2} \rightarrow x\right) \\
& =\Delta(a \rightarrow x)-(a \rightarrow x) \otimes 1-1 \otimes(a \rightarrow x) .
\end{aligned}
$$

Therefore, $a \rightarrow x \in P(H, K)$, and $P(H, K)$ is a $B_{2}$-submodule of $H$. In addition, (2.6) holds for $a \in B_{2}$, as well. The same argument as in the first step, but with $\bar{G}_{1}$ replaced by $\bar{G}_{2}$, proves the desired result of this step.

Putting forward the steps we see finally that $B_{s}(=K)$ trivially acts on $P(H, K)$, as desired.

\section{Proof of Theorem 0.1}

Suppose that we are in the situation of the theorem. The equivalence (ii) $\Longleftrightarrow$ (iii) follows by Proposition 1.1 (1). Obviously, (ii) $\Longrightarrow$ (i). We see that (ii) $\Longrightarrow$ (iv), as in the proof of (e) $\Longrightarrow$ (a) in Remark 1.4 To complete the proof we will show that (i) $\Longrightarrow$ (ii), (iv) $\Longrightarrow$ (ii).

By base extension we may suppose $k=\bar{k}$. Assume (i) or (iv). Whichever is assumed, $H$ does not contain any non-zero primitive $z$ with $z^{p}=0$, since every primitive then needs to be semisimple, by [Mo, 3.2.3]; as for (iv), see Remark 1.4 (a) $\Longrightarrow$ (e). Let $K \subset H$ be a commutative semisimple Hopf subalgebra of the largest dimension. Then, $K \cong k^{G}$, where $G$ is a finite $p$-group, and Lemma 2.2 can apply to $K \subset H$. We wish to prove $K=H$. Suppose $K \subsetneq H$, on the contrary. By Proposition 1.3 (3), we have an element $x$ in $P(H, K) \backslash K$. Then, $K$ and $x$ generate a sub-bialgebra, necessarily a Hopf subalgebra, say $J$, in $H$. By Lemma 2.2. $J$ is 
a (commutative) $K$-algebra generated by $x$, properly including $K$. Keep in mind that by the commutativity, the formula $(\alpha+\beta)^{p^{i}}=\alpha^{p^{i}}+\beta^{p^{i}}$ holds in $J$ and in $J \otimes J$. We wish to prove that $J$ is semisimple, from which we will conclude that $K=H$, as desired.

Let $Q=J / K^{+} J$ denote the quotient Hopf algebra of $J$ divided by $K^{+} J$. Let $\pi: J \rightarrow Q$ denote the quotient map. We regard $J$ as a right $Q$-comodule algebra with respect to $(\operatorname{id} \otimes \pi) \circ \Delta$. Then the $Q$-coinvariants in $J$ are precisely $K$, that is,

$$
K=\{h \in J \mid(\text { id } \otimes \pi) \circ \Delta(h)=h \otimes 1\} .
$$

Set $y=\pi(x)$. Then, $y$ is a non-zero primitive and generates $Q$. A defining relation of $Q$ is given by an equation such as

$$
c_{0} y+c_{1} y^{p}+\cdots+c_{r-1} y^{p^{r-1}}+y^{p^{r}}=0 \quad\left(r>0, c_{i} \in k\right) .
$$

See (1.2). Define

$$
a:=c_{0} x+c_{1} x^{p}+\cdots+c_{r-1} x^{p^{r-1}}+x^{p^{r}} \quad(\in H) .
$$

Then, $\pi(a)=0$, and $a \in P(H, K)$ since now $x^{p^{i}} \in P(H, K)$. Thus, $\Delta(a) \in$ $a \otimes 1+1 \otimes a+K^{+} \otimes K^{+}$. It follows that $a$ is right $Q$-coinvariant, whence $a \in$ $K$. We may suppose $a=0$ by replacing $x$ with $x-b$, where $b \in K^{+}$such that $c_{0} b+c_{1} b^{p}+\cdots+b^{p^{r}}=a$. It then follows by [Mo, 7.2.2, p. 106] that $y \mapsto x$ defines a $Q$-colinear $K$-algebra isomorphism $K \otimes Q \cong J$. Therefore, it suffices to prove that $Q$ is semisimple, or equivalently that $c_{0} \neq 0$. Suppose $c_{0}=0$, on the contrary. The element $z$ in $J \cap P(H, K)$, which is defined by the same formula as (2.9), is again a non-zero primitive with $z^{p}=0$. Here, to see that $z$ is a primitive, notice that the element $\gamma_{z}$ in $K \otimes K\left(=k^{G \times G}\right)$, as defined by (2.5), is zero since $\gamma_{z}^{p}=0$. We have thus proved $c_{0} \neq 0$, as desired.

Remark 3.1. For the condition (iv), we have actually used the condition (c) in Proposition 1.3. Therefore the proof above, applied to the special case $H=u(L)$, proves (c) $\Longleftrightarrow(\mathrm{d}) \Longleftrightarrow$ (e) in the proposition.

Remark 3.2. Suppose $\operatorname{ch} k=p>0$ and that $k$ is algebraically closed. The coordinate Hopf algebra $\mathcal{O}\left(\boldsymbol{\alpha}_{p}\right)$ of the finite group scheme $\boldsymbol{\alpha}_{p}$ is given by $k[z] /\left(z^{p}\right)$ with $z$ a primitive; this is self-dual. Demazure and Gabriel's result [DG, IV, §3, 3.7] cited in the Introduction is translated into the language of Hopf algebras, as follows:

If $H$ is a commutative finitely generated Hopf algebra such that (a) $H$ does not contain any non-trivial idempotent, and (b) $H$ does not have any quotient Hopf algebra isomorphic to $\mathcal{O}\left(\boldsymbol{\alpha}_{p}\right)$, then $H \cong k G$, a group algebra of some (necessarily abelian) group $G$.

By using the same idea as Sweedler's [Sw2, we will deduce this result from our theorem, (iv) $\Longrightarrow$ (iii). It suffices to prove that every finite-dimensional subcoalgebra $C \subset H$ is spanned by grouplikes. For every $n \geq 0$, the ideal $I_{n}$ generated by $h^{p^{n}}\left(h \in H^{+}\right)$is a Hopf ideal. One sees easily that the Hopf algebra $H / I_{n}$ is finite-dimensional and local. The dual $\left(H / I_{n}\right)^{*}$ is irreducible, and by the assumption (b), it does not contain any primitive $z \neq 0$ with $z^{p}=0$. By our theorem, (iv) $\Longrightarrow$ (iii), $H / I_{n}$ is spanned by grouplikes. One sees that $I_{n} \subset\left(H^{+}\right)^{p^{n}}$, and $\bigcap_{n}\left(H^{+}\right)^{n}=0$ follows by the assumption (a) and the Krull intersection theorem; see [Sw2, Theorem 2.10]. Hence, $C \subset H / I_{n}$ for $n \gg 0$, which implies that $C$ is spanned by grouplikes, as desired. 
On the other hand, Sweedler's theorem [Sw2, Theorem 4.1] easily follows from that theorem specialized when $k=\bar{k}$, and the Hopf algebra in question is finitely generated; see also [Mo, Sect. 5.7]. The specialized theorem is formulated just as above, except that the assumption (b) is replaced by

$\left(\mathrm{b}^{\prime}\right) H$ is cosemisimple.

For the result thus formulated, the proof above is valid since by $\left(\mathrm{b}^{\prime}\right), H / I_{n}$ is necessarily cosemisimple, as was proved by Sweedler [Sw2, Corollary 1.10], whence it is spanned by grouplikes, by our theorem, (i) $\Longrightarrow$ (iii) (applied to $\left.\left(H / I_{n}\right)^{*}\right)$.

\section{REFERENCES}

[DG] M. Demazure and P. Gabriel, "Groupes Algébriques", North-Holland, Amsterdam, 1970. MR0302656 (46:1800)

[EG] P. Etingof and S. Gelaki, On finite-dimensional semisimple and cosemisimple Hopf algebras in positive characteristic, Internat. Math. Res. Notices, 1998, No. 16, 851-864. MR1643702 (99i:16068)

[H] G. Hochschild, Representations of restricted Lie algebras of characteristic $p$, Proc. Amer. Math. Soc. 5(1954), 603-605. MR0066361 (16:562d)

[L] R. G. Larson, Characters of Hopf algebras, J. Algebra 17(1971), 352-368. MR0283054 $(44: 287)$

[LR] R. G. Larson and D. E. Radford, Semisimple cosemisimple Hopf algebras, Amer. J. Math. 110(1988), 187-195. MR926744 (89a:16011)

[M] A. Masuoka, Classification of semisimple Hopf algebras, in: M. Hazewinkel (ed.), "Handbook of Algebra", Vol. 5, Elsevier, 2008, pp. 429-455.

[Mo] S. Montgomery, "Hopf Algebras and Their Actions on Rings", CBMS Regional Conference Series in Math., Vol. 82, Amer. Math. Soc., Providence, RI, 1993. MR1243637 (94i:16019)

[SF] H. Strade and R. Farnsteiner, "Modular Lie Algebras and Their Representations", Monogr. Textbooks Pure Appl. Math., Vol. 116, Marcel Dekker, New York and Basel, 1988. MR929682 (89h:17021)

[Sw1] M. Sweedler, "Hopf Algebras", Benjamin, New York, 1969. MR0252485 (40:5705)

[Sw2] M. Sweedler, Connected fully reducible affine group schemes in positive characteristic are abelian. J. Math. Kyoto Univ., 11(1970), 51-70. MR0280499 (43:6219)

Institute of Mathematics, University of Tsukuba, Ibaraki 305-8571, Japan

E-mail address: akira@math.tsukuba.ac.jp 